\title{
Environment-assisted quantum transport and trapping in dimers
}

\author{
Oliver Mülken and Tobias Schmid \\ Physikalisches Institut, Universität Freiburg, Hermann-Herder-Straße 3, 79104 Freiburg, Germany
}

(Dated: March 1, 2022)

\begin{abstract}
We study the dynamics and trapping of excitations for a dimer with an energy off-set $\Delta$ coupled to an external environment. Using a Lindblad quantum master equation approach, we calculate the survival probability $\Pi(t)$ of the excitation and define different lifetimes $\tau_{s}$ of the excitation, corresponding to the duration of the decay of $\Pi(t)$ in between two predefined values. We show that it is not possible to always enhance the overall decay to the trap. However, it is possible, even for not too small environmental couplings and for values of $\Delta$ of the order $\mathcal{O}(1)$, to decrease certain lifetimes $\tau_{s}$, leading to faster decay of $\Pi(t)$ in these time intervals: There is an optimal environmental coupling, leading to a maximal decay for fixed $\Delta$.
\end{abstract}

PACS numbers: $05.60 . \mathrm{Gg}, 05.60 . \mathrm{Cd}, 71.35 .-\mathrm{y}$

\section{INTRODUCTION}

Recent years have seen a growing interest of different communities in coherent energy transfer. For instance, it was noted that in the light-harvesting process of photosynthesis quantum mechanical features of the transfer of excitations (excitons) created by the incoming solar photons might play an important role for the transfer's efficiency [1, 2]. Models of the (coherent) exciton dynamics in the light-harvesting complex of the photosynthetic unit show that the environment does not necessarily destroy all coherent features - even at ambient temperature - but also can support the coherent transfer of excitations [3-7]. In particular, Rebentrost et al. have given a detailed analysis of excitonic dynamics in the Fenna-Matthews-Olsen complex introducing the concept of environment-assisted quantum transport [8]. They showed that the maximum transport efficiency is reached for decoherence rates comparable to the difference between the onsite and the coupling energies. Cao and Silbey showed that exciton trapping can be optimized by suitable choices of, e.g., decoherence and trapping rates [9].

Rapid experimental advances in ultra-cold gases allow to control atoms to a large extent. This offers the possibility to study coherent transport and the effect of environmental changes (e.g., an increase in the temperature or of an external field). Rydberg gases turn out to be very well suited to study the dynamics of excitations [10-12]. At ultra-low temperatures the dynamics in an ensemble of atoms can be efficiently modeled by continuous-time quantum walks [13]. By properly adjusting specific single Rydberg atoms, the moving excitation can be absorbed by these atoms [14, 15]. An analogous process is found in the light-harvesting process, where the exciton eventually will reach the reaction center, where the exciton's energy gets absorbed and converted to chemical energy. In both cases, the decay of the probability $\Pi(t)$ not to get absorbed monitors the excitation's dynamics. If the process is purely coherent, $\Pi(t)$ shows distinct quantum-mechanical features such as a power-law decay in certain, experimentally relevant time-intervals [13, 14].

Since usually the systems (light-harvesting complexes or Rydberg gases) are not isolated from their environment, we will model the dynamics by the Lindblad quantum master equation (LQME) for the reduced density operator of the sys- tem. However, it should be noted that this approach is only valid in a limited parameter range of the coupling to the environment [16]. Our model system is a dimer, represented by two coupled two-level systems, one of which acting as trap. We note that various systems, e.g., with radial symmetry and a trap in the center [17] or with strong coupling between all nodes and weak coupling to the trap, can be mapped onto the dimer.

\section{MODEL}

In general, we consider a small system $S$ (network) of $N$ nodes coupled to an environment. Each node of the network $S$ represents a single two-level system. The basis states $|j\rangle$ which are associated with excitations localized at the nodes $j=1, \ldots, N$ span the Hilbert space of $S$ alone. The Hamiltonian of the total system, $\boldsymbol{H}_{\text {tot }}$, comprises three parts: the Hamiltonians $\boldsymbol{H}_{S}$ for the network $S, \boldsymbol{H}_{R}$ for the environment (reservoir), and $\boldsymbol{H}_{S R}$ for the coupling between system and environment: $\boldsymbol{H}_{\text {tot }}=\boldsymbol{H}_{S}+\boldsymbol{H}_{R}+\boldsymbol{H}_{S R}$.

Within a phenomenological approach, the Hamiltonian $\boldsymbol{H}_{S}$, which incorporates trapping of excitations at the nodes $m \in \mathcal{M}, \mathcal{M} \subset\{1, \ldots, N\}$, is given by $\boldsymbol{H}_{S} \equiv \boldsymbol{H}_{0}-i \boldsymbol{\Gamma}$, where $\boldsymbol{H}_{0}$ is the network Hamiltonian without any trapping and $i \boldsymbol{\Gamma} \equiv i \Gamma \sum_{m}|m\rangle\langle m|(\Gamma>0)$ is the trapping operator, see Ref. [14] for details.

Now, the dynamics of the density operator of the total system is governed by the Liouville-von Neumann equation. Integrating out the environmental degrees of freedom and assuming bilinear couplings between system and environment and the Markov approximation lead to [18]

$$
\dot{\boldsymbol{\rho}}(t)=-i\left[\boldsymbol{H}_{0}, \boldsymbol{\rho}(t)\right]-\{\boldsymbol{\Gamma}, \boldsymbol{\rho}(t)\}+\mathcal{D}[\boldsymbol{\rho}(t)],
$$

where $\mathcal{D}[\boldsymbol{\rho}(t)]$ is responsible for all decoherence effects.

Under certain conditons, such as a weak coupling between the system and the environment, Eq. (1) leads to the Lindblad quantum master equation (LQME), where the Lindblad operators, acting on the Hilbert space of $S$, mimick the influence of the environment on the dynamics [18]. Considering only localized initial conditions $\boldsymbol{\rho}_{k}(0)=|k\rangle\langle k|$ and Lindblad operators in the form $\sqrt{\lambda}|j\rangle\langle j|$ which act only on the diagonal 
elements of $\boldsymbol{\rho}(t)$, the LQME reads [16]

$$
\begin{aligned}
\dot{\boldsymbol{\rho}}_{k}(t)= & -i\left[\boldsymbol{H}_{0}, \boldsymbol{\rho}_{k}(t)\right]-\left\{\boldsymbol{\Gamma}, \boldsymbol{\rho}_{k}(t)\right\} \\
& -2 \lambda \sum_{j=1}^{N}\left(\boldsymbol{\rho}_{k}(t)-\left\langle j\left|\boldsymbol{\rho}_{k}(t)\right| j\right\rangle\right)|j\rangle\langle j|,
\end{aligned}
$$

where the initial condition is labeled at the reduced density operator by the subscript $k$. The parameter $\lambda$ represents the "strength" of the coupling to the environment. Now, the transition probabilities $\pi_{k, j}(t)$ from node $j$ to node $k$ follow from the diagonal elements of $\boldsymbol{\rho}_{k}(t)$, i.e., $\pi_{k, j}(t)=\left\langle j\left|\boldsymbol{\rho}_{k}(t)\right| j\right\rangle$.

In general, the mean survival probability is defined as

$$
\Pi(t) \equiv\left\langle\operatorname{tr}\left[\boldsymbol{\rho}_{k}(t)\right]\right\rangle_{k}=\frac{1}{N-M} \sum_{k \neq m} \sum_{j=1}^{N}\left\langle j\left|\boldsymbol{\rho}_{k}(t)\right| j\right\rangle,
$$

where $\langle\cdot\rangle_{k}$ denotes the average over all possible initial nodes, i.e., all nodes but the trap nodes. Note, that Eq. (3) slightly deviates from the definition used in [14] for the purely coherent case; there, only final nodes $j \neq m$ have been considered. Equation (3) also accounts for the fact that an excitation has a probability of going from a node $m$ to a node $j \neq m$. Thus, $1-\Pi(t)$ describes the total probability of energy dissipation up to time $t$.

\section{RESULTS}

In order to obtain results which are not blurred by the networks complexity, we consider a dimer model, which is composed out of two nodes, one of which acts as the trap. However, it is also possible to map more complex networks onto an effective dimer: One example is a network where the couplings $V_{N}$ between the non-trap nodes are very strong and only a single node is coupled to the trap with a coupling $V \ll V_{N}$, see Fig. 11 or [19]. Another example is a ring of nodes which are all (weakly) coupled to a trap in the center of the ring; if the initial excitation is fully delocalized over the ring, the effective dynamics can also be modeled by a dimer: the non-trap node of the dimer is given by a superposition of all the nodes in the ring and the trap of the dimer is identified with the trap in the center of the ring.
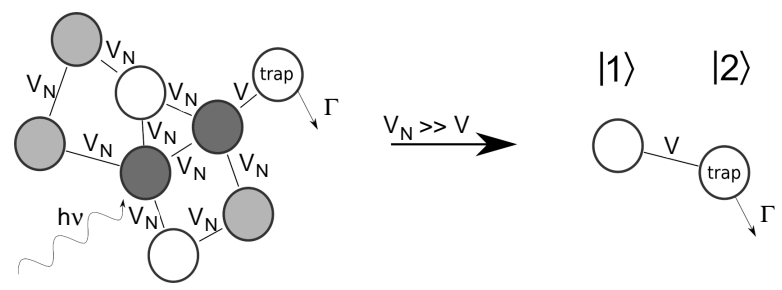

FIG. 1: Schematic illustration of the dimer model.

Now, consider a dimer coupled to an external bath. The Hamiltonian of the dimer without any coupling to the surroundings can be expressed in matrix notation by

$$
\boldsymbol{H}_{S}=\boldsymbol{H}_{0}-i \boldsymbol{\Gamma}=\left(\begin{array}{cc}
E_{1} & -V \\
-V & E_{2}-i \Gamma
\end{array}\right)
$$

where $E_{1}$ and $E_{2}$ are the on-site energies and $V$ is the coupling between the two nodes. Note that in the following we will express all varying parameters in units of $V$. The eigenvalues of $\boldsymbol{H}$ are $E_{ \pm}=E_{1} \pm V e^{ \pm \phi_{\Delta}}$, where $\phi_{\Delta}=$ $\operatorname{arcsinh}[(\Delta-i \Gamma) / 2 V]$ and $\Delta=\left|E_{1}-E_{2}\right|$, and where we assumed $\Gamma \leq 2 V$, such that (for $\Delta=0$ ) the dimer is not overdamped [16]. The bi-orthonormalized eigenstates of $\boldsymbol{H}$ are of the form

$$
\left|\Phi_{ \pm}\right\rangle \equiv \frac{1}{\sqrt{2 \cosh \phi_{\Delta}}}\left(\begin{array}{c}
e^{ \pm \phi_{\Delta} / 2} \\
\pm e^{\mp \phi_{\Delta} / 2}
\end{array}\right)
$$

and

$$
\left|\tilde{\Phi}_{ \pm}\right\rangle \equiv \frac{1}{\sqrt{2 \cosh \phi_{\Delta}^{*}}}\left(\begin{array}{c}
e^{\mp \phi_{\Delta}^{*} / 2} \\
\pm e^{ \pm \phi_{\Delta}^{*} / 2}
\end{array}\right)
$$

where $\phi_{\Delta}^{*}$ is the complex conjugate of $\phi_{\Delta}$. In the limit $\Delta \rightarrow 0$ we recover the results of [16]. Note also, that our model is similar to the one studied by Cao and Silbey in [9]. There, the authors obtained analytical estimates for the (quantum) mean first passage time.
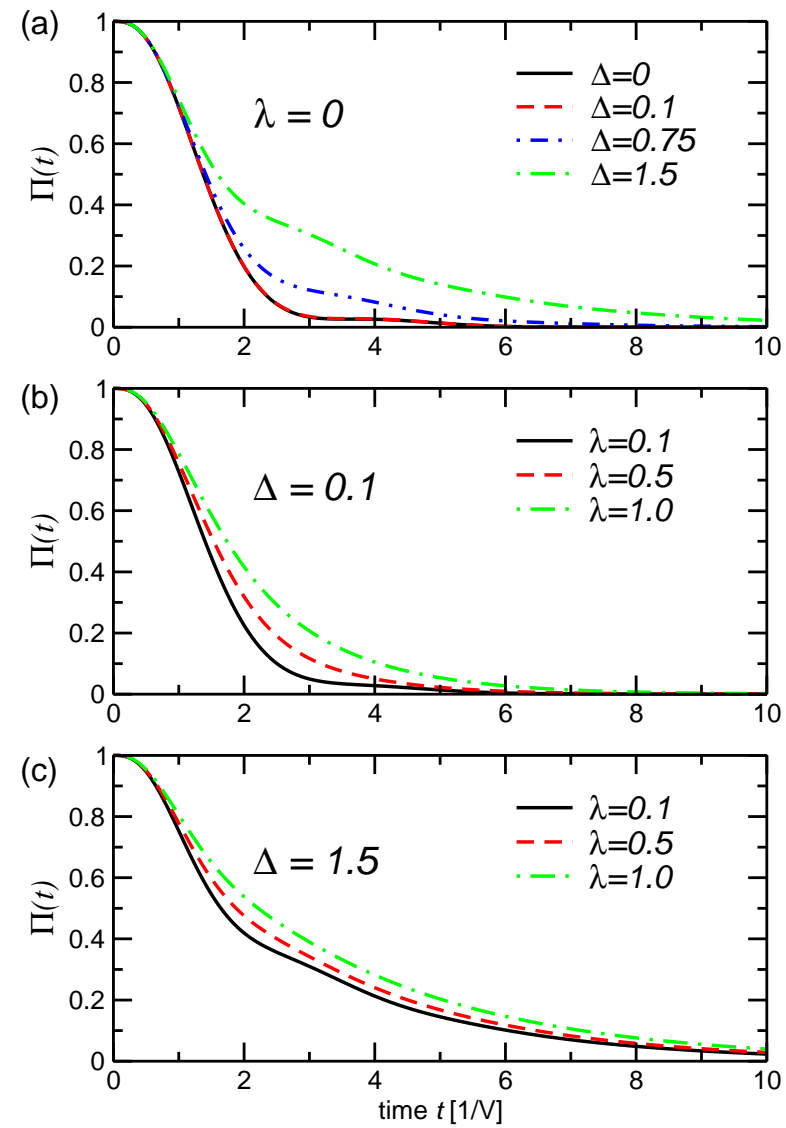

FIG. 2: (Color online) Survival probability $\Pi(t)$ for $\Gamma=V=1$ : (a) for $\lambda=0$ and different $\Delta$, see Eq. (7). (b) solution of the LQME for $\Delta=0.1$ and different $\lambda$. (c) same as (b) but for $\Delta=1.5$. 


\section{A. Survival probabilities}

Let the initial excitation now be placed on the non-trap node 1, i.e., $\left\langle 1\left|\rho_{1}(0)\right| 1\right\rangle=1$. From Eq. (3), the survival probability follows as $\Pi(t)=\left\langle 1\left|\rho_{1}(t)\right| 1\right\rangle+\left\langle 2\left|\rho_{1}(t)\right| 2\right\rangle$. Without dephasing $(\lambda=0)$ the survival probability is obtained by direct diagonalization of $\boldsymbol{H}_{S}$, yielding the analytical result

$$
\begin{aligned}
\Pi(t)= & \frac{e^{-\Gamma t}}{\left|\cosh \left(\phi_{\Delta}\right)\right|^{2}}\left[\left|\cosh \left(t \cdot f(\Gamma, \Delta, V)+\phi_{\Delta}\right)\right|^{2}\right. \\
& \left.+|\sinh (t \cdot f(\Gamma, \Delta, V))|^{2}\right],
\end{aligned}
$$

with $f(\Gamma, \Delta, V)=\frac{1}{2}(\sqrt{(r-y) / 2}+i \sqrt{(r+y) / 2})$, where $r=\sqrt{y^{2}+4 \Gamma^{2} \Delta^{2}}$ and $y=4 V^{2}-\Gamma^{2}+\Delta^{2}$. The limit $\Delta \rightarrow 0$ yields [16]:

$\Pi(t)=\frac{e^{-\Gamma t}}{\cos ^{2}\left(\phi_{\Gamma}\right)}\left[\cos ^{2}\left(V t \cos \left(\phi_{\Gamma}\right)-\phi_{\Gamma}\right)+\sin ^{2}\left(V t \cos \left(\phi_{\Gamma}\right)\right)\right]$,

with $\phi_{\Gamma}=\arcsin (\Gamma / 2 V)$. Letting now also $\Gamma \rightarrow 0$, one arrives at the usual resonant Rabi oscillations for the dimer [20].

Figure 2 a) shows $\Pi(t)$ for $\lambda=0$ and for different values of $\Delta$ : Increasing $\Delta$ leads to a slower decay of $\Pi(t)$. While small values $\Delta=0.1$ do not lead to strong deviations from the $\Delta=0$ results, larger values of $\Delta$ clearly shift the whole $\Pi(t)$ curve upwards. This can be interpreted as the rudimentary onset of localization, the disorder being the off-set $\Delta$. Note also that all curves have a plateau-like region where the slope decreases. By increasing $\Delta$ this "plateau" is shifted to larger values of $\Pi(t)$. This plateau is due to the fact that the dimer only has two nodes. For larger networks, the averaging in $\Pi(t)$ diminishes such effects.

Now, consider the case with a small trapping strength $(\Gamma \ll$ $V)$ and let also the coupling be small $(\lambda \ll V)$. By combining the results for $\lambda=0$ and for $\Gamma=0$, and by expanding all terms except exponentials to first order in $\Gamma$ and $\lambda$, results in the simple exponential decay $\Pi(t) \approx e^{-\Gamma t}$ for not too short times.

Plots of $\Pi(t)$ for different values of $\Delta \neq 0$ and $\lambda \neq 0$ are shown in Fig. 2(b) and (c). The behavior is similar both for small values $\Delta=0.1$ and larger values $\Delta=1.5$ : An increase of the coupling $\lambda$ leads always to a slower decay of $\Pi(t)$. Moreover, for fixed $\lambda$, increasing $\Delta$ also leads to a slower decay of $\Pi(t)$, compare, e.g., the solid curves in Fig. 2(b) and (c). However, both for small and for larger $\Delta$, the curves do not stay equidistant at all times. Therefore, on different time scales the trapping efficiency, here defined as the time it takes to decrease $\Pi(t)$ from one value to another, can vary.

\section{B. Lifetimes}

In order to obtain a quantitative measure for the efficiency of the transport from node 1 to the trap node 2 , we define different lifetimes of the excitation, based on the decay of $\Pi(t)$ between two given values:

$$
\begin{aligned}
& \tau_{1}: \text { decrease of } \Pi(t) \text { from } 1 \text { to } e^{-1}, \\
& \tau_{2}: \text { decrease of } \Pi(t) \text { from } e^{-1} \text { to } e^{-2}, \\
& \tau_{3}: \text { decrease of } \Pi(t) \text { from } e^{-2} \text { to } e^{-3} .
\end{aligned}
$$

see also Fig. 3 Thus, after $t_{1} \equiv \tau_{1}$ about $63 \%$ of the probability has been absorbed by the trap, after $t_{2} \equiv \tau_{1}+\tau_{2}$ about $86 \%$, and after $t_{3} \equiv \tau_{1}+\tau_{2}+\tau_{3}$ about $95 \%$. By considering the lifetimes $\tau_{s}$ individually, we are able to quantify the rate $1 / \tau_{s}$ at which a certain amount of probability gets to be absorbed given the initial value of $\Pi\left(t_{s-1}\right)$.

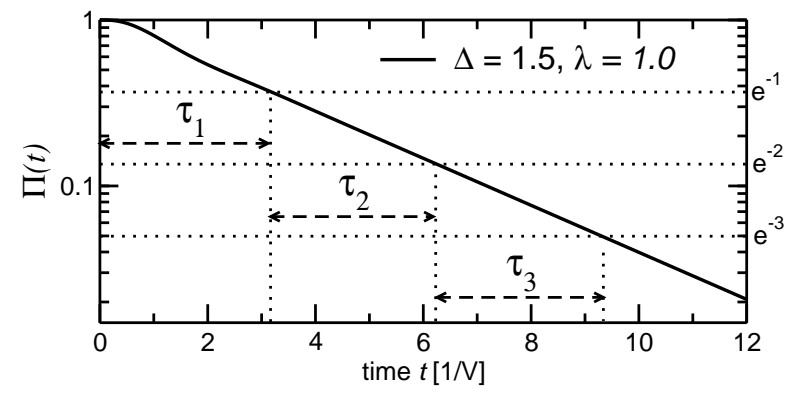

FIG. 3: Definition of the lifetimes $\tau_{1}, \tau_{2}$, and $\tau_{3}$, see text.

Additionally, we also consider the time $\tau_{\infty}$, which is obtained by rewriting the LQME, Eq. (2), in the form $\dot{\vec{\rho}}_{k}(t)=$ $\mathcal{L} \overrightarrow{\boldsymbol{\rho}}_{k}(t)$, whose formal solution is $\overrightarrow{\boldsymbol{\rho}}_{k}(t)=\exp (\mathcal{L} t) \overrightarrow{\boldsymbol{\rho}}_{k}(0)$, where $\overrightarrow{\boldsymbol{\rho}}_{k}(t)=\left(\left\langle 1\left|\boldsymbol{\rho}_{k}(t)\right| 1\right\rangle,\left\langle 1\left|\boldsymbol{\rho}_{k}(t)\right| 2\right\rangle, \ldots,\left\langle N\left|\boldsymbol{\rho}_{k}(t)\right| N\right\rangle\right)$ is an $N^{2}$-dimensional vector. Diagonalization of $\mathcal{L}$ then leads to $\overrightarrow{\boldsymbol{\rho}}_{k}(t)=\mathcal{Q} \exp (\boldsymbol{\Lambda} t) \mathcal{Q}^{-1} \overrightarrow{\boldsymbol{\rho}}_{k}(0)$, where $\mathcal{Q}$ is the eigenvector matrix of $\mathcal{L}$ and $\Lambda$ a diagonal matrix whose elements are the eigenvalues of $\mathcal{L}$. The long-time decay rate $1 / \tau_{\infty}$ is then given by the smallest of all the real parts of the eigenvalues of $\mathcal{L}$. We checked numerically that in our case $\sum_{n} \int_{0}^{\infty} d t\left\langle n\left|\rho_{k}(t)\right| n\right\rangle$ is identical to the results of Cao and Silbey for the mean first passage time, see Eq. (4) in [9]. We note, however, that here we focus on the different lifetimes defined by the mean survival probability and not on averaged quantities as the mean first passage time.

Figures 4(a)-(d) show contour plots of $\tau_{1}, \tau_{2}, \tau_{3}$, and $\tau_{\infty}$, respectively, as functions of $\lambda$ and $\Delta$ for $V=\Gamma=1$. All figures show a similar behavior:

(i) For fixed $\lambda$ and increasing $\Delta$ all lifetimes increase monotonically (except $\tau_{3}$ for small $\lambda$, see below).

(ii) For not too small values of $\Delta$, however, all lifetimes except $\tau_{1}$ (see below) first decrease with increasing $\lambda$ to a minimum and then increase again.

The latter behavior is exemplified in Fig. 4(e) for $\Delta=1.5$. Clearly there is a minimum for $\tau_{2}, \tau_{3}$, and $\tau_{\infty}$ at about the same value of $\lambda \approx 0.5$. Therefore, there is an optimal value of $\lambda \neq 0$ which leads to larger rates $1 / \tau_{s}(s \neq 1)$ compared to the ones for $\lambda=0$. This implies, for instance, that for $\tau_{2}$ an additional $23 \%$ of the probability gets to be absorbed by the trap faster if the dimer is coupled to the environment. However, it does not neccessarily imply that $86 \%$ of the probability transferred to the trap after time $t_{2}$ gets to be absorbed faster. 
(a)

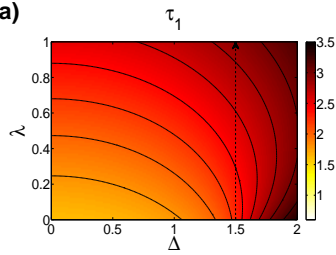

(c)

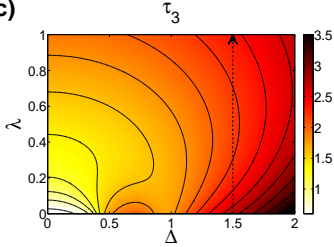

(b)

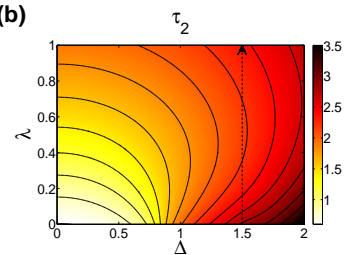

(d)

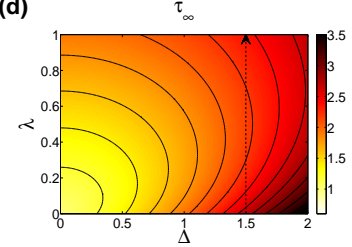

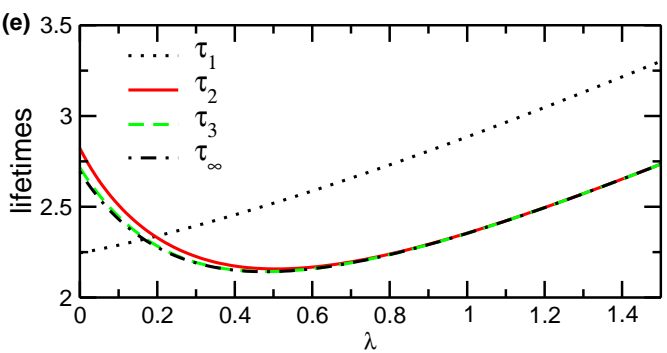

FIG. 4: (Color online) (a)-(d) Contour plots of the lifetimes $\tau_{1}, \tau_{2}$, $\tau_{3}$, and $\tau_{\infty}$, respectively, as functions of $\lambda$ and $\Delta$ for $V=\Gamma=1$. The arrows at $\Delta=1.5$ show the location of the cuts for fixed $\Delta$ and increasing $\lambda$ displayed panel (e).

The same reasoning holds also for $\tau_{3}$ and $\tau_{\infty}$. The initial absorption at the rate $1 / \tau_{1}$ cannot be enhanced for these values of $\Delta$ of the order $\mathcal{O}(1)$ by the coupling to the environment. This would mean that, in the interval $\tau_{1}$, (the later part of) a $\Pi(t)$ curve for $\lambda^{\prime}>\lambda$ would lie below the curve for $\lambda$, which within our LQME approach is not the case, see Figs. 22(b) and (c). However, increasing $\Delta$ to larger values also leads to a shallow minimum for $\tau_{1}$ as a function of $\lambda$, see Fig. 4(a) for $\Delta \approx 2$.

The increase of the rates also depends on the value of $\Delta$. While an increase of $\tau_{\infty}$ is visible even for small $\Delta \approx 0.1$, this is not the case for $\tau_{2}$ and $\tau_{3}$. Thus, in order to obtain a sufficiently large increase of the rates $\tau_{2}$ and $\tau_{3}$, one needs to have a value of $\Delta$ of the order $\mathcal{O}(1)$. Intuitively, one observes a "competition" between localization and decoherence effects: While the energy offset $\Delta$ tends to bind the excitation at the initial node, the environmental coupling $\lambda$ tends to

spread the excitation evenly over the dimer. However, with increasing values of $\lambda$ one approaches the quantum Zeno limit which also leads to a slower decay of the survival probability and thus to larger lifetimes. Therefore, the minimal lifetimes can be viewed as being caused by an optimal decoherence rate $\lambda$ destroying localization due to $\Delta$. We note that our results are in agreement with the findings of Cao and Silbey [9] and with the ones of Rebentrost et al. [8].

The fact that the lifetimes $\tau_{s}(s \geq 2)$ lie on the same curve for $\lambda \gtrsim 0.5$ translates to an (on average) exponential decrease of the survival probability: Let the lifetimes $\tau_{s}$ be obtained piecewise, approximately via $\Pi\left(t_{s-1}\right)-\Pi\left(t_{s}\right) \sim \exp \left(-a \tau_{s}\right)$, where $a$ is some constant. Now, if $\tau_{s}=\tau$ for all $s$, we obtain $\Pi\left(t_{s-r}\right)-\Pi\left(t_{s}\right) \sim \exp (-a r \tau)$, with $r=1, \ldots, s-1$. Allowing also $r=s$, one has $\Pi\left(t_{s}\right) \sim \Pi\left(t_{0}\right)-\exp \left(-a t_{s}\right)$, where $t_{s}=s \tau$.

Finally, we remark on one peculiar feature of the behavior of $\tau_{3}$. For small values of $\lambda \lesssim 0.1$, the lifetime $\tau_{3}$ has a local maximum at $\Delta \approx 0.7$, visible as a small "lagoon" in Fig. 4(c). This is due to the plateau of $\Pi(t)$, mentioned above. By increasing $\Delta$ for fixed (small) values of $\lambda$ the plateau is shifted to larger values of $\Pi(t)$ and, therefore, can eventually lie in the region between $\Pi(t)=e^{-1}$ and $\Pi(t)=e^{-2}$. This then might lead to longer lifetimes $\tau_{3}$. The other lifetimes $\tau_{2}$ and $\tau_{\infty}$ are not affected by this.

\section{CONCLUSIONS}

In conclusion, we have studied the trapping of excitations for a dimer with an energy off-set and with environmental coupling. The survival probability, $\Pi(t)$, not to get trapped (obtained from a Lindblad quantum master equation) shows distinct features depending on the strength $\lambda$ of the coupling to the environment and on the energy off-set $\Delta$ : While it is not possible to always enhance the overall efficiency of the decay to the trap, it is possible to increase the rate of trapping in certain intervals of time, leading to faster decay of probability in these intervals. The fastest decay is obtained for an optimal value of $\lambda$ which is about half the value of the coupling $V$ between the nodes. However, a substantial increase is only obtained for values of $\Delta$ of the order $\mathcal{O}(1)$.

Acknowledgemetns. - Support from the Deutsche Forschungsgemeinschaft (DFG) and the Fonds der Chemischen Industrie is gratefully acknowledged. We thank Alexander Blumen for continuous support and fruitful discussions.
[1] G. S. Engel, T. R. Calhoun, R. L. Read, T.-K. Ahn, T. Manal, Y.-C. Cheng, R. E. Blankenship, and G. R. Fleming, Nature 446, 782 (2007).

[2] E. Collini, C. Y. Wong, K. E. Wilk, P. M. G. Curmi, P. Brumer, and G. D. Scholes, Nature 463, 644 (2010).

[3] M. Mohseni, P. Rebentrost, S. Lloyd, and A. Aspuru-Guzik, The Journal of Chemical Physics 129, 174106 (2008).

[4] F. Caruso, A. W. Chin, A. Datta, S. F. Huelga, and M. B. Plenio, The Journal of Chemical Physics 131, 105106 (2009).
[5] A. Olaya-Castro, C. F. Lee, F. F. Olsen, and N. F. Johnson, Phys. Rev. B 78, 085115 (2008).

[6] Y. C. Cheng and R. J. Silbey, Phys. Rev. Lett. 96, 028103 (2006).

[7] M. Thorwart, J. Eckel, J. Reina, P. Nalbach, and S. Weiss, Chem. Phys. Lett. 478, 234 (2009).

[8] P. Rebentrost, M. Mohseni, I. Kassal, S. Lloyd, and A. AspuruGuzik, New. J. Phys. (2009).

[9] J. Cao and R. J. Silbey, J. Phys. Chem. A 113, 13825 (2009). 
[10] W. R. Anderson, J. R. Veale, and T. F. Gallagher, Phys. Rev. Lett. 80, 249 (1998).

[11] I. Mourachko, C. Comparat, F. de Tomasi, A. Fioretti, P. Nosbaum, V. Akulin, and P. Pillet, Phys. Rev. Lett. 80, 253 (1998).

[12] S. Westermann, T. Amthor, A. L. de Oliveira, J. Deiglmayr, M. Reetz-Lamour, and M. Weidemüller, Eur. Phys. J. D 40, 37 (2006).

[13] O. Mülken and A. Blumen, Physica E 42, 576 (2010).

[14] O. Mülken, A. Blumen, T. Amthor, C. Giese, M. Reetz-Lamour, and M. Weidemüller, Phys. Rev. Lett. 99, 090601 (2007).

[15] M. Reetz-Lamour, T. Amthor, J. Deiglmayr, and M. Weidemüller, Phys. Rev. Lett. 100, 253001 (2008).

[16] O. Mülken, L. Mühlbacher, T. Schmid, and A. Blumen, Phys. Rev. E 81, 041114 (2010).

[17] O. Mülken, V. Bierbaum, and A. Blumen, J. Chem. Phys. 124,
124905 (2006).

[18] H.-P. Breuer and F. Petruccione, The Theory of Open Quantum Systems (Oxford University Press, Oxford, England, 2006).

[19] H. v. Amerongen, L. Valkunas, and R. v. Grondelle, Photosynthetic Excitons (World Scientific Publishing Co., Singapore, 2000).

[20] J. Sakurai, Modern Quantum Mechanics (Addison-Wesley, Redwood City, CA, 1994), 2nd ed.

[21] J. Adolphs and T. Renger, Biophys. J. 91(8), 2778 (2006).

[22] S. I. E. Vulto, M. A. de Baat, R. J. W. Louwe, H. P. Permentier, T. Neef, M. Miller, H. v. Amerongen, and T. J. Aartsma, J. Phys. Chem. B 102, 9577 (1998).

[23] V. May and O. Kühn, Charge and energy transfer dynamics in molecular systems (Wiley-VCH, Weinheim, 2004). 